\title{
Groupwise density and related cardinals
}

\author{
Andreas Blass \\ Mathematics Department, University of Michigan, Ann Arbor, MI 48109, USA \\ Received August 14, 1989
}

\begin{abstract}
We prove several theorems about the cardinal $g$ associated with groupwise density. With respect to a natural ordering of families of nondecreasing maps from $\omega$ to $\omega$, all families of size $<\mathrm{g}$ are below all unbounded families. With respect to a natural ordering of filters on $\omega$, all filters generated by $<\mathfrak{g}$ sets are below all non-feeble filters. If $\mathfrak{u}<\mathfrak{g}$ then $\mathfrak{b}=\mathfrak{u}$ and $\mathfrak{g}=\mathfrak{b}=\mathfrak{c}$. (The definitions of these cardinals are recalled in the introduction.) Finally, some consequences deduced from $\mathrm{u}<\mathfrak{g}$ by Laflamme are shown to be equivalent to $\mathfrak{u}<\mathfrak{g}$.
\end{abstract}

\section{Introduction}

Groupwise dense families and the associated cardinal number $g$ were introduced in [5]. (These and other concepts used here will be defined later in this introduction.) Their original purpose was to provide a succinct formulation, namely the cardinal inequality $\mathfrak{u}<\mathfrak{g}$ where $\mathfrak{u}$ is the minimum number of generators for an ultrafilter, of the crucial combinatorial properties obtained by a forcing construction due to Shelah $[3,4]$. Thus, consistency proofs using Shelah's models can be carried out without referring directly to the models, by deducing the desired statements from $\mathfrak{u}<\mathfrak{g}$.

In view of the origin of $g$, it is not surprising that most research about $g$ has involved deducing consequences from $u<\mathrm{g}$. See $[5,6,11,12]$; the main exception seems to be Theorem 2 of [6].

In the first two sections of this paper, we shall prove some results about $g$ that do not involve $\mathfrak{u}$. The proofs of the results are based on the same ideas as previous work that assumed $\mathfrak{u}<\mathfrak{g}$, but we believe it is worthwhile to free these methods from this connection with $\mathfrak{u}$ in order to bring out more clearly the role of $g$. The main results here, Theorems 2 and 5, say that certain structures that are quite trivial in cardinalities $<\mathfrak{b}$ (the bounding number) are nearly (but not quite) as simple in cardinalities $<\mathfrak{g}$. Of course, these results are interesting only if $\mathfrak{b}<\mathfrak{g}$, which seems to be a weaker hypothesis than $\mathfrak{u}<\mathfrak{g}$. 
In Sect. 3, we revert to the pattern of earlier research and deduce consequences from $\mathfrak{u}<\mathrm{g}$. The consequences (Theorems 6, 7, and 8) all have the form that certain cardinals must be equal, specifically $\mathfrak{b}=\mathfrak{u}$ and $\mathfrak{g}=\boldsymbol{D}=\boldsymbol{c}$. As a corollary, we obtain that two combinatorial results that were deduced from $\mathfrak{u}<\mathfrak{g}$ in [12] are in fact equivalent to $\mathfrak{u}<\mathfrak{g}$.

The remainder of this introduction will be devoted to setting out the notation and terminology that we shall use. $[\omega]^{\omega}$ is the set of all infinite subsets of the set $\omega$ of natural numbers. For $A \in[\omega]^{\omega}$ and $n \in \omega$, next $(A, n)$ is the smallest number $\geqq n$ in A. $\omega \nearrow \omega$ is the set of non-decreasing functions from $\omega$ to $\omega$. We say that a function $f$ (eventually) majorizes a function $g$ if $f(n) \geqq g(n)$ for all (sufficiently large) $n \in \omega$; (eventual) majorization on an infinite $A \subseteq \omega$ has the same definition except that $n$ ranges only over $A$. A function from $\omega$ to $\omega$ is bounded if it is majorized by a constant function. A family $\mathscr{X}$ of functions dominates another family $\mathscr{Y}$ if each $g \in \mathscr{Y}$ is eventually majorized by some $f \in \mathscr{X}$. Filters are always assumed to be proper filters on $\omega$ and to contain all cofinite sets; thus ultrafilters are non-principal. For any family $\mathscr{X}$ of subsets of $\omega$ and any $f: \omega \rightarrow \omega$, we define

$$
f(\mathscr{X})=\left\{Z \subseteq \omega \mid f^{-1}(Z) \in \mathscr{X}\right\} .
$$

Notice that, if $\mathscr{F}$ is a filter and $f$ is finite-to-one, then $f(\mathscr{F})$ is the filter generated by the sets $f(X)$, where $X$ ranges over any base for $\mathscr{F}$.

$\mathfrak{c}$ is the cardinality of the continuum. $\mathfrak{b}$ is the minimum cardinality of a family $\subseteq \omega \nearrow \omega$ that is not dominated by a single function. $D$ is the minimum cardinality of a family $\subseteq \omega \nearrow \omega$ that dominates $\omega \nearrow \omega . \mathfrak{u}$ is the minimum cardinality of any ultrafilter base. (These four cardinals and our notation for them are from [7].)

A family $\mathscr{G} \subseteq[\omega]^{\omega}$ is groupwise dense if

(a) whenever $X \in \mathscr{G}$ and $Y \in[\omega]^{\omega}$ and $Y-X$ is finite, then $Y \in \mathscr{G}$, and

(b) for any infinite family of disjoint, nonempty, finite subsets of $\omega$, the union of some infinite subfamily is in $\mathscr{G}$.

It is easy to check that, in the presence of (a), the family in (b) can be assumed to be a partition of $\omega$ into finite intervals. We shall usually use (b) in this form. Notice that, if $\Pi$ and $\Pi^{\prime}$ are partitions of $\omega$ into finite pieces and if every piece of $\Pi^{\prime}$ is a union of pieces of $\Pi$, then (b) for $\Pi$ follows trivially from (b) for $\Pi^{\prime}$. Thus, when checking that (b) holds for a given partition $\Pi$, we may consider instead of $\Pi$ any $\Pi^{\prime}$ obtained by merging pieces of $\Pi$.

$\mathfrak{g}$ is the smallest cardinal $\kappa$ such that some $\kappa$ groupwise dense families have no common member.

The results in this paper, except for Theorem 8 and its corollary, were presented at the Oberwolfach meeting on set theory in January, 1989. The paper was written during a visit to the University of Heidelberg. I thank Professor G.H. Müller for arranging this visit and for his warm hospitality.

\section{1. $g$ is almost $b$ for families of functions}

In this section, we consider families $\mathscr{X}$ of non-decreasing functions from $\omega$ to $\omega$, i.e., $\mathscr{X} \subseteq \omega \nearrow \omega$, and to avoid trivialities we consider only families that contain at least one unbounded function. A pre-ordering relation $\leqq$ between such families was introduced in [8] (motivated by an application in abelian group theory) and 
studied further in $[9,2,5,12]$ under various set-theoretic hypotheses. The definition of $\mathscr{X} \leqq \mathscr{Y}$ is that there exists $r \in \omega \nearrow \omega$ such that each $f \in \mathscr{X}$ is eventually majorized by some function $g \circ r$ with $g \in \mathscr{Y}$. (This is obviously reflexive, and to prove transitivity just compose the $r$ 's.) We shall give an alternate description of $\leqq$ which may make it seem more natural, and then we use this description to simplify the proof of a result (alluded to by the title of the section) that was proved but not stated in [5] and stated but not proved in [12].

For families $\mathscr{X}, \mathscr{Y} \subseteq \omega \nearrow \omega$, we say that $\mathscr{X}$ is somewhere dominated by $\mathscr{Y}$ if there is an infinite set $A \cong \omega$ such that each $f \in \mathscr{X}$ is eventually majorized on $A$ by some $g \in \mathscr{Y}$.

Theorem 1. The relation $\leqq$ is the transitive closure of the relation "is somewhere dominated by".

Proof. If $\mathscr{X}$ is somewhere dominated by $\mathscr{Y}$, with $A \subseteq \omega$ as witness, then, for each $f \in \mathscr{X}$ there is $\mathrm{g} \in \mathscr{Y}$ such that, for all sufficiently large $n$,

$$
f(n) \leqq f(\operatorname{next}(A, n)) \leqq g(\operatorname{next}(A, n)),
$$

so $\mathscr{X} \leqq \mathscr{Y}$ with witness given by $r(n)=\operatorname{next}(A, n)$. As $\leqq$ is transitive, all that remains to be proved is that, if $\mathscr{X} \leqq \mathscr{Y}$, then $\mathscr{X}$ is somewhere dominated by $\mathscr{Z}_{1}$, which is somewhere dominated by $\mathscr{Z}_{2}, \ldots$, which is somewhere dominated by $\mathscr{Z}_{n}$, which is somewhere dominated by $\mathscr{Y}$. In fact, we shall do this with only a single $\mathscr{Z}$.

Assume $\mathscr{X} \leqq \mathscr{Y}$ with witness $r$. Choose $a_{0}<a_{1}<a_{2}<\ldots$ inductively so that $a_{0}=0$ and $r\left(a_{n}\right)<a_{n+1}$ for all $n$. For each $g \in \mathscr{Y}$, define $\hat{g}$ by

$$
\hat{g}(x)=g\left(a_{n+1}-1\right) \quad \text { if } x \in\left[a_{n}, a_{n+1}\right) ;
$$

thus $\hat{g}$ takes, throughout any interval $\left[a_{n}, a_{n+1}\right)$, the largest value taken by $g$ on that interval. Let $\mathscr{Z}=\{\hat{g} \mid g \in \mathscr{Y}\}$. Since $\hat{g}$ and $g$ agree at the right end of each interval, $\mathscr{Z}$ is somewhere dominated by $\mathscr{Y}$ with witness $\left\{a_{n+1}-1 \mid n \in \omega\right\}$. We complete the proof by checking that $\mathscr{X}$ is somewhere dominated by $\mathscr{Z}$, with witness $\left\{a_{n} \mid n \in \omega\right\}$. For each $f \in \mathscr{X}$, there is $g \in \mathscr{Y}$ such that $f(n) \leqq g(r(n))$ for all sufficiently large $n$. Thus, for large $n$, we have

$$
f\left(a_{n}\right) \leqq g\left(r\left(a_{n}\right)\right) \leqq g\left(a_{n+1}-1\right)=\hat{g}\left(a_{n}\right) .
$$

As $\hat{\mathrm{g}} \in \mathscr{Z}$, the proof is complete.

It is easy to see that, if $\mathscr{X}$ is dominated by some $h \in \omega>\omega$, then $\mathscr{X} \leqq \mathscr{Y}$ for any $\mathscr{Y}$. Indeed, if $g$ is any unbounded function in $\mathscr{Y}$, then $r$ can be chosen so that $g \circ r$ majorizes $h$, hence dominates $\mathscr{X}$. It is also easy to see that any $\mathscr{X}$ that is $\leqq$ a dominated $\mathscr{Y}$ must itself be dominated (by $h \circ r$ if $h$ dominates $\mathscr{Y}$ and $r$ witnesses $\mathscr{X} \leqq \mathscr{Y}$ ). Passing to equivalence classes with respect to the equivalence relation induced by $\leqq$, and thus viewing $\leqq$ as a partial order, we have that the dominated families constitute the smallest element in this order. (Had we not required every family to contain an unbounded function, there would have been an $(\omega+1)$ sequence of lower elements.)

Any $\mathscr{X}$ of cardinality $<\mathfrak{b}$ is, by the preceding remarks, at the bottom of our order. The next theorem and its corollary show that any $\mathscr{X}$ of cardinality $<\mathrm{g}$ is very near the bottom; it is $\leqq$ every $\mathscr{Y}$ except possibly the dominated $\mathscr{Y}$ 's. Of course 
this is of interest only when $\mathfrak{g}>\mathfrak{b}$. The corollary appeared as Lemma 9 in [12], with a reference to [5] for the proof. The proof to be given below is simpler than that in [5] $((0) \rightarrow(1)$ in Theorem 1$)$ and gives the slightly stronger result in the theorem rather than only the corollary.

Theorem 2. If $|\mathscr{X}|<\mathrm{g}$ and $\mathscr{Y}$ is not dominated, then $\mathscr{X}$ is somewhere dominated by $\mathscr{Y}$.

Proof. For each $f \in \mathscr{X}$, let

$$
\mathscr{G}_{f}=\left\{A \in[\omega]^{\omega} \mid(\exists g \in \mathscr{Y}) g \text { eventually majorizes } f \text { on } A\right\} .
$$

Any $A$ that belongs to all the $\mathscr{G}_{f}$ 's simultaneously can serve as a witness that $\mathscr{X}$ is somewhere dominated by $\mathscr{Y}$. So, as there are fewer than $g$ families $\mathscr{G}_{f}$, we need only show that each $\mathscr{G}_{f}$ is groupwise dense; indeed, we need only check condition (ii) in the definition of groupwise density, as (i) is trivial. So let $f \in \mathscr{X}$ be given and let $\omega$ be partitioned into intervals $\left[a_{0}, a_{1}\right),\left[a_{1}, a_{2}\right), \ldots$ Define

$$
f^{\prime}(n)=f\left(a_{k+2}\right) \quad \text { if } n \in\left[a_{k}, a_{k+1}\right) .
$$

As $\mathscr{Y}$ is not dominated, fix some $g \in \mathscr{Y}$ such that $g(n) \geqq f^{\prime}(n)$ for infinitely many $n$. For each such $n$, if $n \in\left[a_{k}, a_{k+1}\right)$, then for any $x$ in the next interval $\left[a_{k+1}, a_{k+2}\right)$ we have

$$
f(x) \leqq f\left(a_{k+2}\right)=f^{\prime}(n) \leqq g(n) \leqq g(x) .
$$

Thus, $g$ majorizes $f$ on $\left[a_{k+1}, a_{k+2}\right)$ whenever some $n \in\left[a_{k}, a_{k+1}\right)$ satisfies $g(n)$ $\geqq f^{\prime}(n)$. So $g$ majorizes $f$ on the union of infinitely many intervals from the given partition, and that means that this union belongs to $\mathscr{G}_{f}$.

Corollary. If $|\mathscr{X}|<\mathfrak{g}$, then $\mathscr{X} \leqq \mathscr{Y}$ for all undominated $\mathscr{Y}$.

If $\mathfrak{b}<\mathfrak{g}$, then there is an undominated family of size $<\mathfrak{g}$, so the corollary tells us that the bottom of the ordering consists of two comparable elements, and everything else is above these.

\section{2. $g$ is almost $b$ for filters}

In this section, we obtain a result analogous to (the corollary of) Theorem 2 , for filters instead of families of functions. First, we need the concepts analogous to dominated families and the ordering in Sect. 1. All filters that we consider will be proper filters on $\omega$ and will contain all cofinite sets.

A filter $\mathscr{F}$ is feeble if there is a finite-to-one $f: \omega \rightarrow \omega$ such that $f(\mathscr{F})$ is the filter of cofinite sets. Equivalently, there is a partition of $\omega$ into finite pieces (namely $f^{-1}\{n\}$ ) such that each set in $\mathscr{F}$ intersects all but finitely many pieces. One can always arrange for the pieces to be intervals (just make sure each interval includes some $f^{-1}\{n\}$ ), i.e., one can arrange for $f$ to be monotone.

The pre-ordering relation that we shall use for filters is defined by putting $\mathscr{F} \leqq \mathscr{H}$ if and only if, for some finite-to-one $f: \omega \rightarrow \omega, f(\mathscr{F}) \subseteq f(\mathscr{H})$. It is not obvious that this is a transitive relation; before we prove that it is, it will be useful to make some preliminary observations.

Our convention that filters must contain all cofinite sets does not prevent $f(\mathscr{F})$ and $f(\mathscr{H})$ from being filters; they contain all cofinite sets because $f$ is finite-to-one. If $\mathscr{F}$ is feeble, then a witness $f$ to this fact also witnesses that $\mathscr{F} \leqq \mathscr{H}$ for every $\mathscr{H}$. 
Also, if $\mathscr{F}$ is feeble then so is $f(\mathscr{F})$ for any finite-to-one $f$; indeed, if the feebleness of $\mathscr{F}$ is witnessed by a partition $\Pi$ of $\omega$ into finite pieces, then the feebleness of $f(\mathscr{F})$ is witnessed by any $\Pi^{\prime}$ such that, for each piece $P \in \Pi^{\prime}, f^{-1}(P)$ includes a piece from $\Pi$, and it is trivial to construct such a $\Pi^{\prime}$. It follows that, if $\mathscr{F}$ is feeble and $\mathscr{K} \leqq \mathscr{F}$ with witness $f$, then $f(\mathscr{K})$ is included in the feeble filter $f(\mathscr{F})$, so $f(\mathscr{K})$ is feeble, and so $\mathscr{K}$ is feeble (compose $f$ with a witness that $f(\mathscr{K})$ is feeble). So any filter $\leqq \mathrm{a}$ feeble one is feeble. Thus, the feeble filters constitute the lowest class in the (alleged) ordering (induced by) $\leqq$.

It will be useful to know that the witnesses $f$ for the relation $\mathscr{F} \leqq \mathscr{H}$ can always be chosen to be monotone. In terms of the partition of $\omega$ into the fibers $f^{-1}\{n\}$ of $f$, to say that $f$ witnesses $\mathscr{F} \leqq \mathscr{H}$ means that, for each $X \in \mathscr{F}$ there is $Y \in \mathscr{H}$ such that every piece meeting $Y$ also meets $X$. Our objective is to show that, if there is such a partition of $\omega$ into finite pieces, then there is one where all the pieces are intervals. We remark that, if a partition $\Pi$ witnesses $\mathscr{F} \leqq \mathscr{H}$ in the sense just described, then so does any $\Pi^{\prime}$ (partition of $\omega$ into finite pieces) whose pieces are unions of pieces of $\Pi$; we say that $\Pi^{\prime}$ is obtained from $\Pi$ by merging pieces.

Given a partition $\Pi$ witnessing that $\mathscr{F} \leqq \mathscr{H}$, construct a partition of $\omega$ into intervals $\left[a_{0}, a_{1}\right),\left[a_{1}, a_{2}\right), \ldots$ such that each piece of $\Pi$ meets at most two of the intervals, and if it meets two then they are consecutive. Such intervals are easy to find inductively; set $a_{0}=0$ and choose $a_{n+1}$ larger than all members of all pieces of $\Pi$ that intersect $\left[0, a_{n}\right)$. Because $\Pi$ witnessed $\mathscr{F} \leqq \mathscr{H}$, we have, for each $X \in \mathscr{F}$, some $Y \in \mathscr{H}$ such that, whenever $Y$ meets one of our intervals, then $X$ meets that interval or one of the two adjacent intervals.

We may (and do) suppose $\mathscr{H}$ is not feeble, for if it were then $\mathscr{F}$ would also be feeble and there would be a monotone $f$ with $f(\mathscr{F})=\{$ all cofinite sets $\} \subseteq f(\mathscr{H})$, as desired. So $\mathscr{H}$ contains a set $Z$ that is disjoint from infinitely many of the intervals $\left[a_{2 n}, a_{2 n+2}\right)$, say all such intervals for $n \in Q$, where $Q$ is an infinite subset of $\omega$. We use the points $a_{2 n+1}$ for $n \in Q$ as the endpoints defining a new partition $\Pi^{\prime}$ of $\omega$ into intervals; that is, if $n<m$ are consecutive in $Q$, then $\left[a_{2 n+1}, a_{2 m+1}\right) \in \Pi^{\prime}$. (We can ignore $\left[0, a_{2 \min (Q)+1}\right)$ or add it to $\Pi^{\prime}$.) We shall see that $\Pi^{\prime}$ witnesses that $\mathscr{F} \leqq \mathscr{H}$.

Let $X \in \mathscr{F}$. As remarked above, there is $Y \in \mathscr{H}$ such that, whenever $Y$ meets an interval $\left[a_{n}, a_{n+1}\right)$ then $X$ meets $\left[a_{n-1}, a_{n+2}\right)$. We also have $Z \in \mathscr{H}$ disjoint from $\left[a_{2 n}, a_{2 n+2}\right)$ for all $n \in Q$. As $\mathscr{H}$ is a filter, it contains a set $W$ (namely the intersection of $Y, Z$, and a cofinite set) having the properties just stated for $Y$ and $Z$ and also being disjoint from $\left[0, a_{2 \min (Q)+1}\right)$. If $W$ meets a piece $\left[a_{2 n+1}, a_{2 m+1}\right)$ of $\Pi^{\prime}$, then this intersection does not occur in $\left[a_{2 n+1}, a_{2 n+2}\right)$ or $\left[a_{2 m}, a_{2 m+1}\right)$ because $W \subseteq Z$, so $W$ and therefore $Y$ must meet $\left[a_{2 n+2}, a_{2 m}\right)$, and therefore $X$ meets $\left[a_{2 n+1}, a_{2 m+1}\right)$. This completes the proof that $\Pi^{\prime}$ witnesses $\mathscr{F} \leqq \mathscr{H}$.

Now that we can use interval partitions to witness the $\leqq$ relation, it is not hard to prove transitivity. Assume $\mathscr{F} \leqq \mathscr{H} \leqq \mathscr{K}$. Notice first that, by our earlier remarks, if any one of these three filters is feeble, then so is $\mathscr{F}$, and thus $\mathscr{F} \leqq \mathscr{K}$ as desired. So assume the three filters are not feeble, and let $\Pi$ and $\Pi^{\prime}$ be partitions into intervals witnessing $\mathscr{F} \leqq \mathscr{H}$ and $\mathscr{H} \leqq \mathscr{K}$ respectively. Let us write $\left[a_{n}, a_{n+1}\right)$ for the intervals in $\Pi$ and $\left[a_{n}^{\prime}, a_{n+1}^{\prime}\right)$ for those in $\Pi^{\prime}$. By merging intervals, we can arrange that $0=a_{0}$ $=a_{0}^{\prime}<a_{1}<a_{1}^{\prime}<a_{2}<a_{2}^{\prime}<\ldots$. As $\mathscr{H}$ isn't feeble, it contains a set $Z$ disjoint from infinitely many pieces of $\Pi$, say $\left[a_{n}, a_{n+1}\right)$ for all $n$ in a certain infinite set $Q \subseteq \omega$. Let $\Pi^{\prime \prime}$ be the partition into intervals whose endpoints are the $a_{n}^{\prime}$ for $n \in Q$. Thus, $\Pi^{\prime \prime}$ is 
obtainable from $\Pi^{\prime}$ by merging intervals, and so $\Pi^{\prime \prime}$ witnesses $\mathscr{H} \leqq \mathscr{K}$. We shall show that the same $\Pi^{\prime \prime}$ also witnesses $\mathscr{F} \leqq \mathscr{H}$. It then follows trivially that $\Pi^{\prime \prime}$ witnesses $\mathscr{F} \leqq \mathscr{K}$, as desired.

To show that $\Pi^{\prime \prime}$ witnesses $\mathscr{F} \leqq \mathscr{H}$, let any $X \in \mathscr{F}$ be given and find $Y \in \mathscr{H}$ such that every interval $\left[a_{n}, a_{n+1}\right)$ meeting $Y$ also meets $X$. Let $W$ be the intersection of this $Y$, the $Z$ from the previous paragraph, and the (cofinite) set of numbers $\geqq a_{\min (Q)}^{\prime}$. Then $W \in \mathscr{H}$, and we complete the proof by showing that any piece of $\Pi^{\prime \prime}$ meeting $W$ also meets $X$. Any such piece has the form $\left[a_{n}^{\prime}, a_{m}^{\prime}\right)$ with $n<m$ in $Q$. This interval is covered by the three intervals $\left[a_{n}, a_{n+1}\right),\left[a_{n+1}, a_{m}\right)$, and $\left[a_{m}, a_{m+1}\right)$, so $W$ meets one of these. It doesn't meet the first or third, by our choice of $Q$ and because $W \subseteq Z$. So $W$ and therefore $Y$ and therefore $X$ must meet $\left[a_{n+1}, a_{m}\right)$, which is included in $\left[a_{n}^{\prime}, a_{m}^{\prime}\right)$. This completes the proof that $\leqq$ is transitive.

It is now legitimate to speak of the partial ordering $\leqq$ of equivalence classes of filters with respect to the equivalence relation induced by the pre-order $\leqq$. The smallest element in this order consists of the feeble filters. Thus, feeble filters play the same role here that dominated families played in Sect. 1. That the analogy is not trivial is indicated by the following two known results.

Theorem 3 (Solomon [16]). Any filter generated by fewer than $\mathfrak{b}$ sets is feeble.

(Solomon's statement of the theorem asserts only that such a filter is not an ultrafilter, but his proof shows that it is feeble.)

Theorem 4 (Simon [15]). There is a non-feeble filter generated by $\mathfrak{b}$ sets.

Thus, the cardinal $b$ plays the same role with respect to the ordering of filters as it does with respect to the ordering of families of functions in the previous section. The following theorem extends the analogy by showing that $g$ also plays the same role with respect to both orderings. Its proof uses the same idea as in [6, Theorem 3].

Theorem 5. Let $\mathscr{F}$ and $\mathscr{H}$ be filters. Assume that $\mathscr{F}$ is not feeble and that $\mathscr{H}$ is generated by a family of fewer than $\mathrm{g}$ sets. Then $\mathscr{H} \leqq \mathscr{F}$.

Proof. Let $\mathscr{B}$ be a family of fewer than $\mathrm{g}$ sets generating $\mathscr{H}$. We may assume that $\mathscr{B}$ is closed under finite intersection, as closing it will not increase its cardinality. So every set in $\mathscr{H}$ has a subset in $\mathscr{B}$. For each $B \in \mathscr{B}$, let

$$
\begin{aligned}
& \mathscr{G}_{B}=\left\{X \in[\omega]^{\omega} \mid(\exists A \in \mathscr{F}) \text { If } x<y \text { are in } X\right. \text { and the interval } \\
& {[x, y) \text { meets } A, \text { then it also meets } B\} . }
\end{aligned}
$$

$\mathscr{G}_{B}$ satisfies part (a) of the definition of groupwise dense, because $\mathscr{F}$ includes all cofinite sets. To see that it also satisfies (b), let a partition of $\omega$ into intervals be given. By merging intervals, we can assume that each interval in the partition contains an element of $B$. As $\mathscr{F}$ is not feeble, there is $A \in \mathscr{F}$ disjoint from infinitely many intervals from the partition. Let $X$ be the union of these intervals. If $x<y$ are in $X$ and $[x, y)$ meets $A$, then this intersection must be in an interval (of our partition) not in $X$, as $X$ is disjoint from $A$. So $[x, y)$ includes this entire interval, which contains an element of $B$. Thus, $X \in \mathscr{G}_{B}$ with witness $A$. As $X$ is a union of pieces of the given partition, we have proved that $\mathscr{G}_{B}$ is groupwise dense. 
As $|\mathscr{B}|<\mathfrak{g}$, there is an $X$ common to all the $\mathscr{G}_{B}$ for $B \in \mathscr{B}$. The intervals $[x, y)$, for $x<y$ consecutive in $X$, (along with $[0, \min (X))$ ) form a partition of $\omega$ witnessing that $\mathscr{H} \leqq \mathscr{F}$. Indeed, if $Q \in \mathscr{H}$ then there is $B \in \mathscr{B}$ with $B \subseteq Q$, and, as $X \in \mathscr{G}_{B}$, there is $A \in \mathscr{F}$ such that, if $[x, y)$ meets $A$ then it also meets $B$, hence also $Q$. (As $\mathscr{F}$ includes the cofinite sets, we can arrange that $A$ does not meet $[0, \min (X))$.) Thus, $\mathscr{H} \leqq \mathscr{F}$.

Like Theorem 2 and its corollary, Theorem 5 is of interest only if $\mathfrak{b}<\mathfrak{g}$, as otherwise the $\mathscr{H}$ in the theorem would be feeble. If $\mathfrak{b}<\mathfrak{g}$, then, by Theorem 4 , the $\mathscr{H}$ in Theorem 5 need not be feeble, so we have that the bottom of the ordering of (equivalence classes of) filters consists of two consecutive elements, and everything else is above these.

If $\mathscr{U}$ is an ultrafilter, then so is $f(\mathscr{U})$ for any finite-to-one $f: \omega \rightarrow \omega$ (indeed, for any $f$ not constant on any set in $\mathscr{U}$ ), so " $f(\mathscr{U}) \subseteq f(\mathscr{F})$ " is synonymous with " $f(\mathscr{U})$ $=f(\mathscr{F})^{\prime}$. That is, $\mathscr{U}$ is maximal with respect to $\leqq$. Thus, the pre-ordering $\leqq$ restricted to ultrafilters is an equivalence relation, namely the relation of cofinal equivalence studied in $[1,2]$.

Conversely, as any filter is included in an ultrafilter, every equivalence class in our ordering is $\leqq$ one containing an ultrafilter. Thus, the maximal elements in our ordering contain ultrafilters and therefore consist of filters that are mapped to ultrafilters by finite-to-one maps.

The preceding discussion re-proves the result from [5] that $\mathfrak{u}<\mathfrak{g}$ implies the following principle of "Filter Dichotomy".

"Every filter can be mapped, by a finite-to-one function, to

either an ultrafilter or the filter of cofinite sets".

This, in turn, implies (as shown also in [5]) the principle of near coherence of filters, NCF, studied in [1-4]:

For all ultrafilters $\mathscr{U}$ and $\mathscr{V}, \mathscr{U} \leqq \mathscr{V}$; in other words, there is only one maximal element in the ordering of equivalence classes of filters.

It remains an open problem whether either of the implications

$$
\mathfrak{u}<\mathfrak{g} \Rightarrow \text { Filter Dichotomy } \Rightarrow \text { NCF }
$$

can be reversed.

\section{The effect of $\mathfrak{u}<\mathfrak{g}$ on other cardinals}

In this section, we consider the three cardinal inequalities

$$
\mathfrak{b} \leqq \mathfrak{u}, \quad \mathfrak{g} \leqq \mathfrak{d}, \quad \text { and } \quad \mathfrak{d} \leqq \mathfrak{c} .
$$

The first of these is due to Solomon [16] (see Theorem 3 above), the second is proved in [6], and the third is trivial. None of these three inequalities is reversible in ZFC. Indeed, all are simultaneously strict in the model obtained from a model of GCH by adding $\aleph_{2}$ Cohen reals and then $\aleph_{3}$ random reals; in this model, $\mathfrak{b}=\mathfrak{g}=\aleph_{1}, \mathfrak{b}=\aleph_{2}$, and $\mathfrak{u}=\mathfrak{c}=\aleph_{3}$. (For $\mathfrak{g}=\aleph_{1}$, see [6, corollary to Theorem 2]. The 
rest of these equations are well known.) We shall show that $u<\mathfrak{g}$ implies that all three inequalities reduce to equalities: $\mathfrak{b}=\mathfrak{u}$ and $\mathfrak{g}=\mathfrak{c}$. (That $\mathfrak{b}$ was mentioned above is not just for decoration. The proof of $g=c$ will consist of separate proofs of $\mathfrak{g}=\mathrm{D}$ and $\mathrm{D}=\mathrm{C}$, one of which can be done with a possibly weaker hypothesis than $\mathfrak{u}<\mathfrak{g}$.)

Theorem 6. Assume $\mathfrak{u}<\mathfrak{g}$ (or just Filter Dichotomy). Then $\mathfrak{b}=\mathfrak{u}$.

Proof. By Theorem 4, there is a non-feeble filter $\mathscr{F}$ generated by $\mathfrak{b}$ sets. By Filter Dichotomy, there is a (finite-to-one) $f$ such that $f(\mathscr{F})$ is an ultrafilter. But $f(\mathscr{F})$ is generated by $\mathfrak{b}$ sets, namely the $f$-images of the generators of $\mathscr{F}$. So $\mathfrak{u} \leqq \mathfrak{b}$. As $\mathfrak{b} \leqq \mathfrak{u}$ always, the theorem is proved.

Theorem 7. Assume $\mathfrak{u}<\mathfrak{g}$ (or just Filter Dichotomy). Then $\mathfrak{d}=\mathrm{c}$.

Proof. Let $\mathscr{D} \subseteq \omega \lambda \omega$ be a family of size $D$ that dominates all of $\omega \lambda \omega$. For each $f \in \mathscr{D}$, construct $A_{f}$ and $B_{f}$ in $[\omega]^{\omega}$ as follows. Inductively choose $a_{0}<a_{1}<a_{2}<\ldots$ so that $a_{0}=0$ and $a_{n+1}>f\left(a_{n}\right)$ for all $n$. Then let $A_{f}=\bigcup_{n \in \omega}\left[a_{4 n}, a_{4 n+1}\right)$ and $B_{f}=\bigcup_{n \in \omega}\left[a_{4 n+2}, a_{4 n+3}\right)$. The crucial property of $A_{f}$ and $B_{f}$ is that no interval of the form $[x, f(x)]$ can meet them both. Indeed, the choice of the $a_{n}$ 's ensures that $[x, f(x)]$ is included in the union of two adjacent intervals of the form $\left[a_{n}, a_{n+1}\right)$, but we never put such an interval into $A_{f}$ and an adjacent one into $B_{f}$.

Now choose, for each $f \in \mathscr{D}$, two ultrafilters $\mathscr{U}_{f}$ and $\mathscr{V}_{f}$ containing $A_{f}$ and $B_{f}$ respectively. Let $\mathscr{F}$ be the intersection of all the $\mathscr{U}_{f}$ 's and $\mathscr{V}_{f}$ 's for all $f \in \mathscr{D}$. We apply Filter Dichotomy to the filter $\mathscr{F}$.

Case 1. $\mathscr{F}$ is feeble.

Fix a finite-to-one $f$ such that $f(\mathscr{F})$ contains only cofinite sets. Also fix a family $\mathscr{X} \subseteq[\omega]^{\omega}$ of $\mathfrak{c}$ almost disjoint sets. (See [10, p. 242] for the existence of such a family.) Almost-disjointness means that, for $X \neq Y$ in $\mathscr{X}, X \cap Y$ is finite, and therefore so is $f^{-1}(X) \cap f^{-1}(Y)$ as $f$ is finite-to-one.

For each $X \in \mathscr{X}$, as $X$ is infinite, $\omega-X \notin f(\mathscr{F})$, so $\omega-f^{-1}(X) \notin \mathscr{F}$. In view of the definition of $\mathscr{F}$, we can assign to each $X \in \mathscr{X}$ an ultrafilter $\mathscr{W}_{X}$, equal to either $\mathscr{U}_{g}$ or $\mathscr{V}_{g}$ for some $g \in \mathscr{D}$, such that $\omega-f^{-1}(X) \notin \mathscr{W}_{X}$ and therefore $g^{-1}(X) \in \mathscr{W}_{X}$. For $X \neq Y$ in $\mathscr{X}, f^{-1}(X)$ and $f^{-1}(Y)$ have finite intersection, so no ultrafilter contains them both; thus $\mathscr{W}_{X} \neq \mathscr{W}_{Y}$. We have, therefore, a one-to-one map $X \mapsto \mathscr{W}_{X}$ from a set $\mathscr{X}$ of cardinality $c$ into a set $\left\{\mathscr{U}_{g}, \mathscr{V}_{g} \mid g \in \mathscr{D}\right\}$ of cardinality $\mathrm{D}$. So $\mathfrak{c} \leqq \grave{d}$. As the reverse inequality is trivial, the theorem is proved in Case 1.

Case 2. $g(\mathscr{F})$ is an ultrafilter, for some finite-to-one $g$.

As $\mathscr{D}$ dominates $\omega \nearrow \omega$, fix an $f \in \mathscr{D}$ such that, for all sufficiently large $n$,

$$
f(n)>\max g^{-1}\{g(n)\}
$$

Thus, for all sufficiently large $k$, the fiber $g^{-1}\{k\}$ is included in $[n, f(n))$ for some $n$ (namely $n=\min g^{-1}\{k\}$, or an arbitrary $n$ if $g^{-1}\{k\}$ is empty). Thus, by the crucial property of $A_{f}$ and $B_{f}$, only finitely many fibers of $g$ meet them both. This means that $g\left(A_{f}\right) \cap g\left(B_{f}\right)$ is finite. As $A_{f} \in \mathscr{U}_{f}$ and $B_{f} \in \mathscr{V}_{f}$, it follows that $g\left(\mathscr{U}_{f}\right)$ and $g\left(\mathscr{V}_{f}\right)$ are distinct. But they both include $g(\mathscr{F})$, an ultrafilter. This is absurd, so Case 2 cannot occur, and so the proof is complete. 
Remark. The argument for Case 1 is essentially the proof in [13] that the intersection of fewer than $c$ ultrafilters cannot have the Baire property, modulo Talagrand's proof [17] that, for filters, the Baire property is equivalent to feebleness. The proof in [13] looks more complicated because the Baire property is used directly.

Theorem 8. Assume $\mathfrak{u}<\mathfrak{g}$. Then $\mathfrak{g}=\mathfrak{d}$.

Proof. As $\mathfrak{g} \leqq \mathrm{D}$ in any case, we need only consider an arbitrary fewer than $\mathrm{D}$ groupwise dense families $\mathscr{G}_{\alpha}$ and find a common member. For each $\alpha$, let

$$
\mathscr{A}_{\alpha}=\left\{X \in[\omega]^{\omega} \mid \omega-X \in \mathscr{G}_{\alpha}\right\} \text {. }
$$

Then each $\mathscr{A}_{\alpha}$ is closed under supersets and finite modifications, so by [12, Theorems 7 and 10] there is a finite-to-one $f_{\alpha}$ such that $f_{\alpha}\left(\mathscr{A}_{\alpha}\right)$ is $[\omega]^{\omega}$ or an ultrafilter or the filter of cofinite sets.

If $f_{\alpha}\left(\mathscr{A}_{\alpha}\right)$ contains only cofinite sets, then, for every infinite $Q \subseteq \omega, \omega-Q \notin f_{\alpha}\left(\mathscr{A}_{\alpha}\right)$, so $\omega-f_{\alpha}^{-1}(Q)=f_{\alpha}^{-1}(\omega-Q) \notin \mathscr{A}_{\alpha}$, and so $f_{\alpha}^{-1}(Q) \notin \mathscr{G}_{\alpha}$. This means that $\mathscr{G}_{\alpha}$ contains no infinite union of fibers of $f_{\alpha}$, which contradicts the groupwise density of $\mathscr{G}_{\alpha}$.

So each $f_{\alpha}\left(\mathscr{A}_{\alpha}\right)$ is either $[\omega]^{\omega}$ or an ultrafilter. In either case, $f_{\alpha}\left(\mathscr{A}_{\alpha}\right)$ includes an ultrafilter $\mathscr{U}_{\alpha}$. These fewer than $\triangleright$ ultrafilters $\mathscr{U}_{\alpha}$ have, according to [1, Theorem 19], a common finite-to-one image $\mathscr{U}$. So, by composing $f_{\alpha}$ with an appropriate finiteto-one map and renaming the result $f_{\alpha}$, we may assume $f_{\alpha}\left(\mathscr{A}_{\alpha}\right) \supseteq \mathscr{U}$ for all $\alpha$. By [1, Theorem 14], additional composing and renaming allows us to assume that $\mathscr{U}$ is generated by fewer than $\delta$ sets.

The complement of the range of $f_{\alpha}$ is certainly not in $f_{\alpha}\left(\mathscr{A}_{\alpha}\right)$, so the range of $f_{\alpha}$ is in $\mathscr{U}$. This means that the following functions are defined for $\mathscr{U}$-almost all $n$, i.e., for all $n$ in a set (which may depend on $\alpha$ ) in $\mathscr{U}$.

$$
\begin{aligned}
\min _{\alpha}(n)=\min \left\{x \mid f_{\alpha}(x)=n\right\} \\
\max _{\alpha}(n)=\max \left\{x \mid f_{\alpha}(x)=n\right\} .
\end{aligned}
$$

We claim that there exist a monotone finite-to-one function $\ell: \omega \rightarrow \omega$ and a strictly monotone function $h: \omega \rightarrow \omega$ such that, for each $\alpha, \mathscr{U}$-almost all $n$ satisfy

$$
\ell(n) \leqq \min _{\alpha}(n) \leqq \max _{\alpha}(n) \leqq h(n) .
$$

In other words, in the ultrapower $\mathscr{U}$-prod $\omega,[\ell]$ is a lower bound for all the $\left[\min _{\alpha}\right]$ 's and $[h]$ is an upper bound for all the $\left[\max _{\alpha}\right]$ 's.

The existence of $[h]$ follows immediately from [1, Theorem 16], which says that (since we have NCF) $\mathscr{U}$-prod $\omega$ has cofinality $\mathfrak{D}$. As there are fewer than $D\left[\max _{\alpha}\right]$ 's, they must have an upper bound $[h]$; by increasing $h$, we can make it strictly monotone.

To obtain $[\ell]$, we first observe that $\mathscr{U}$ is not rapid. Indeed, rapidity would mean that the enumerating functions

$$
n \mapsto n^{\text {th }} \text { element of } A,
$$

as $A$ ranges over a basis of $\mathscr{U}$, constitute a dominating family, but $\mathscr{U}$ has a basis of cardinality $<\mathbf{b}$. Using an alternate characterization of rapidity due to Puritz [14, p. 733], we find that there is a finite-to-one monotone function $\ell: \omega \rightarrow \omega$ such that $[\ell]$ is below $[f]$ in $\mathscr{U}$-prod $\omega$ for all $f$ that are one-to-one on a set in $\mathscr{U}$. Since $\min _{\alpha}$ is one-to-one on range $\left(f_{\alpha}\right)$, this $[\ell]$ is as desired. 
(As Puritz does not give a proof of the result quoted above, we provide one. Suppose there is no $\ell$ with the required property. Let $g: \omega \rightarrow \omega$ be strictly increasing. Define $\ell(x)=$ the smallest $y$ with $g(y)>x$. By assumption, there is a set $A \in \mathscr{U}$ and there is a one-to-one function $f: A \rightarrow \omega$ such that $f(x)<\ell(x)$ for all $x \in A$. Then, on the first $n$ elements of $A, f$ takes $n$ different values, so at least one of them is $\geqq n-1$. So $\ell$ also takes a value $\geqq n-1$ at one of the first $n$ elements of $A$, hence at the $n^{\text {th }}$ element of $A$ (as $\ell$ is monotone). So, if $x$ is the $n^{\text {th }}$ element of $A$, then, by definition of $\ell, g(n-2) \leqq x$. This means that the enumerating function of $A$ minus its first two elements majorizes $g$. As $g$ is arbitrary, $\mathscr{U}$ is rapid.)

Inductively define, using $\ell$ and $h$ as above, a sequence $a_{0}<a_{1}<a_{2}<\ldots$ by setting $a_{0}=0$ and choosing $a_{n+1}$ so large that $\ell\left(a_{n+1}\right)>h\left(a_{n}\right)$. Let $X=\left\{h\left(a_{n}\right) \mid n \in \omega\right\}$.

We shall show that, for each $\alpha$, there exists a set $D_{\alpha} \in \mathscr{U}$ such that,

$$
\text { if } x, y \in X \text { and } f_{\alpha}(x)=f_{\alpha}(y) \in D_{\alpha} \text {, then } x=y \text {. }
$$

In other words, $f_{\alpha}$ is one-to-one on $X$ insofar as its values are in $D_{\alpha}$. The required set $D_{\alpha}$ is simply the set in $\mathscr{U}$ on which the inequalities $\ell(n) \leqq \min _{\alpha}(n)$ and $\max _{\alpha}(n) \leqq h(n)$ hold. To see that this works, suppose $x<y$ in $X$ and $f_{\alpha}(x), f_{\alpha}(y) \in D_{\alpha}$; we shall show $f_{\alpha}(x)<f_{\alpha}(y)$. By definition of $X$, we have $x=h\left(a_{j}\right)$ and $y=h\left(a_{k}\right)$ for some $j<k$. Combining this with the definitions of $\min _{\alpha}$ and $\max _{\alpha}$ and with the meaning of $f_{\alpha}(x), f_{\alpha}(y) \in D_{\alpha}$, we find that

and

$$
h\left(a_{k}\right)=y \leqq \max _{\alpha}\left(f_{\alpha}(y)\right) \leqq h\left(f_{\alpha}(y)\right)
$$

$$
\ell\left(a_{j+1}\right)>h\left(a_{j}\right)=x \geqq \min _{\alpha}\left(f_{\alpha}(x)\right) \geqq \ell\left(f_{\alpha}(x)\right) .
$$

As $h$ is strictly monotone, we have $a_{k} \leqq f_{\alpha}(y)$. As $\ell$ is monotone, we have $a_{j+1}>f_{\alpha}(x)$. But $j<k$, so

$$
f_{\alpha}(x)<a_{j+1} \leqq a_{k} \leqq f_{\alpha}(y) .
$$

This completes the proof that $f_{\alpha}$ is one-to-one on $X$ insofar as its values lie in $D_{\alpha}$.

Let $\mathscr{Y}$ be a family of $\mathrm{c}$ almost disjoint infinite subsets of $X$. By the claim just proved, for each $\alpha$ the sets $f_{\alpha}(Y) \cap D_{\alpha}$ for $Y \in \mathscr{Y}$ are almost disjoint, so at most one of them is in $\mathscr{U}$. As there are $c Y Y$ 's and fewer than $D$ (hence certainly fewer than $c$ ) $\alpha$ 's, there must be at least one $Y \in \mathscr{Y}$ (in fact $c$ of them) such that $f_{\alpha}(Y) \cap D_{\alpha} \notin \mathscr{U}$ for any $\alpha$. Fix such a $Y$.

For each $\alpha$, we have $f_{\alpha}(Y) \cap D_{\alpha} \notin \mathscr{U}$, but $D_{\alpha} \in \mathscr{U}$, so $f_{\alpha}(Y) \notin \mathscr{U}$, and therefore $\omega-f_{\alpha}(Y) \in \mathscr{U}$. As $\mathscr{U} \subseteq f_{\alpha}\left(A_{\alpha}\right)$ we conclude that $\omega-f_{\alpha}^{-1}\left(f_{\alpha}(Y)\right)=f_{\alpha}^{-1}\left(\omega-f_{\alpha}(Y)\right) \in \mathscr{A}_{\alpha}$, and therefore, by definition of $\mathscr{A}_{\alpha}, f_{\alpha}^{-1}\left(f_{\alpha}(Y)\right) \in \mathscr{G}_{\alpha}$. Finally, as $\mathscr{G}_{\alpha}$ is closed under subsets, $Y \in \mathscr{G}_{\alpha}$. Thus, we have a set $Y$ belonging to all of the given $\mathscr{G}_{\alpha}^{\text {'s. }}$

In [12], Laflamme deduced from $\mathfrak{u}<\mathfrak{g}$ that

the ordering of (equivalence classes of) subsets of $\omega \nearrow \omega$ in Sect. 1 is a chain of length 5 .

From this, he deduced the fact, used near the beginning of the proof of Theorem 8 , that

(B) every upward closed subset of $[\omega]^{\omega}$ is sent, by some finite-toone function $\omega \rightarrow \omega$, to $[\omega]^{\omega}$ or an ultrafilter or a family of cofinite sets. 
The proof of Theorem 8 allows us to complete the cycle of implications.

Corollary. $\mathfrak{u}<\mathfrak{g}$ is equivalent to each of (A) and (B).

Proof. In view of Laflamme's results, we need only deduce $\mathfrak{u}<\mathfrak{g}$ from (B). So assume (B). As the image of a filter under a (finite-to-one) map is a filter and not $[\omega]^{\omega}$, we have Filter Dichotomy and therefore NCF. All uses of $\mathfrak{u}<\mathrm{g}$ in the proof of Theorem 8 were uses of NCF, except for one use of (B). So our present assumption (B) allows us to carry out the same proof and conclude $\mathfrak{g}=\mathrm{D}$. But, as we have NCF, [1, Corollary 15] gives us $\mathfrak{u}<\mathfrak{b}$. Therefore $\mathfrak{u}<\mathfrak{g}$.

\section{References}

1. Blass, A.: Near coherence of filters. I. Cofinal equivalence of models of arithmetic. Notre Dame J. Formal Logic 27, 579-591 (1986)

2. Blass, A.: Near coherence of filters. II. Applications to operator ideals, the Stone-Čech remainder of a half-line, order ideals of sequences, and slenderness of groups. Trans. Am. Math. Soc. 300, 557-581 (1987)

3. Blass, A., Shelah, S.: There may be simple $P_{\mathrm{N}_{1}}$ and $P_{\mathrm{N}_{2}}$ points and the Rudin-Keisler order may be downward directed. Ann. Pure Appl. Logic 83, 213-243 (1987)

4. Blass, A., Shelah, S.: Near coherence of filters. III. A simplified consistency proof. Notre Dame J. Formal Logic 30, 530-538 (1989)

5. Blass, A., Laflamme, C.: Consistency results about filters and the number of inequivalent growth types. J. Symb. Logic 54, 50-56 (1989)

6. Blass, A.: Applications of superperfect forcing and its relatives. In: Steprano, J., Watson, S. (eds.) Set theory and its applications. (Lect. Notes Math. vol. 1401, pp. 18-40) Berlin Heidelberg New York: Springer 1989

7. van Douwen, E.: The integers and topology. In: Kunen, K., Vaughan, J. (eds.) Handbook of set-theoretic topology. Amsterdam: North-Holland 1984, pp. 111-167

8. Göbel, R., Wald, B.: Wachstumstypen und schlanke Gruppen. Symp. Math. 23, 201-239 (1979)

9. Göbel, R., Wald, B.: Martin's axiom implies the existence of certain slender groups. Math. Z. 172, 107-121 (1980)

10. Jech, T.: Set theory. New York: Academic Press 1978

11. Just, W., Laflamme, C.: Classification of measure zero sets with respect to their open covers. (To appear in Trans. Am. Math. Soc.)

12. Laflamme, C.: Equivalence of families of functions on the natural numbers. (To appear)

13. Plewik, S.: Intersections and unions of ultrafilters without the Baire property. Bull. Pol. Acad. Sci. Math. 35, 805-808 (1987)

14. Puritz, C.: Ultrafilters and standard functions in non-standard arithmetic. Proc. Lond. Math. Soc. III. Ser. 22, 705-733 (1971)

15. Simon, P.: Private communication. August 1987

16. Solomon, R.: Families of sets and functions. Czech. Math. J. 27, 556-559 (1977)

17. Talagrand, M.: Filtres non mesurables et compacts de fonctions mesurables. Stud. Math. 67, 13-43 (1980) 